\title{
Polycystic kidney disease in patients on the renal transplant waiting list: trends in hematocrit and survival Kevin C Abbott ${ }^{* 1}$ and Lawrence Y Agodoa ${ }^{2}$
}

Address: ${ }^{1}$ Nephrology Service, Walter Reed Army Medical Center, Washington, D.C., and Uniformed Services University of the Health Sciences, Bethesda, MD and ${ }^{2}$ NIDDK, NIH, Bethesda, MD

E-mail: Kevin C Abbott* - kevin.abbott@na.amedd.army.mil; Lawrence Y Agodoa - agodoal@extra.niddk.nih.gov

*Corresponding author permitted in all media for any non-commercial purpose, provided this notice is preserved along with the article's original URL.

Keywords: Polycystic kidney disease, Caucasian, female, EPO, peritoneal dialysis, transplantation, complications, dialysis, USRDS, age, albumin, hemoglobin, weight, dysrythmias, mortality, frequency

\begin{abstract}
Background: The patient characteristics and mortality associated with autosomal dominant polycystic kidney disease (PKD) have not been characterized for a national sample of end stage renal disease (ESRD) patients on the renal transplant waiting list.
\end{abstract}

Methods: 40,493 patients in the United States Renal Data System who were initiated on ESRD therapy between I April 1995 and 29 June 1999 and later enrolled on the renal transplant waiting list were analyzed in an historical cohort study of the relationship between hematocrit at the time of presentation to ESRD and survival (using Cox Regression) in patients with PKD as a cause of ESRD.

Results: Hematocrit levels at presentation to ESRD increased significantly over more recent years of the study. Hematocrit rose in parallel in patients with and without PKD, but patients with PKD had consistently higher hemoglobin. PKD was independently associated with higher hematocrit in multiple linear regression analysis $(p<0.000 \mathrm{I})$. In logistic regression, higher hematocrit was independently associated with PKD. In Cox Regression analysis, PKD was associated with statistically significant improved survival both in comparison with diabetic (hazard ratio, $0.64,95 \%$ $\mathrm{Cl} 0.53-0.77, \mathrm{p}<0.00 \mathrm{I})$ and non-diabetic (HR $0.68,95 \% \mathrm{Cl} 0.56-0.82, \mathrm{p}=0.00 \mathrm{I})$ ESRD patients, adjusted for all other factors.

Conclusions: Hematocrit at presentation to ESRD was significantly higher in patients with PKD compared with patients with other causes of ESRD. The survival advantage of PKD in ESRD persisted even adjusted for differences in hematocrit and in comparison with patients on the renal transplant waiting list.

\section{Background}

Polycystic kidney disease (PKD) has recently been associ- ated with decreased mortality compared with non-diabetic end stage renal disease (ESRD) patients. [1] Because 
Hematocrit levels have been reported to be higher in PKD patients than in other patients with ESRD, it has been suggested that the survival of these patients may be related to their elevated Hematocrit levels. [2] Another recent study adjusted for the level of Hematocrit at initiation of dialysis, and found the survival benefit of PKD persisted. [3] However, because PKD patients are much more likely to be placed on the renal transplant waiting list and receive renal transplantation than other ESRD patients [1,3], it is possible this survival advantage may also be due to their younger age and better general health. It would be useful to know if the differences in Hematocrit and survival persisted in analysis limited to patients enrolled on the renal transplant waiting list. Therefore, we analyzed data from the standard analysis files of the 2000 United States Renal Data System (USRDS) database. Our objectives were to analyze differences in Hematocrit and hemoglobin between patients with PKD and other causes of ESRD, whether these differences have changed over time, and whether this or other factors are associated with their relative survival advantage in ESRD.

\section{Methods}

We analyzed a national registry (the 2000 USRDS) in an historical cohort study of the association of Polycystic kidney disease with Hematocrit and patient survival among patients enrolled on the renal transplant waiting list. Information on comorbidity, as well as height and weight to calculate BMI, was obtained from the USRDS file SAF.MEDEVID. This file is derived from the Center for Medicare and Medicaid Studies (formerly HCFA) medical evidence form (2728) starting with a sample of ESRD patients prior to April 1995 and universal afterward, and has been validated for use in research. [4] We first selected patients who initiated ESRD therapy between 1 April 1995 to 29 June 1999 who had data sufficient to calculate hematocrit. From this cohort, we selected patients enrolled on the renal transplant waiting list, excluding any dates of listing prior to 1 April 1995. The date patients on the renal transplant waiting list first received a renal transplant was also extracted, also excluding any dates prior to 1 April 1995. Recipients of organs other than kidneys and of transplants without preceding dialysis were excluded. The variables included in the USRDS standard analysis files (SAF's), as well as data collection methods and validation studies, are listed at the USRDS website [www.usrds.org], under 'Researcher's Guide to the USRDS Database', Section E, 'Contents of all the SAF's' (Standard Analysis Files), and published in the USRDS. The demographics of the end stage renal disease population have been previously described (2001 USRDS report). Dialysis patients younger than age 65 are eligible for Medicare 90 days after starting dialysis, with a waiver granted to those choosing home therapies. Therefore, hospitalization and mortality data may be incomplete during the first 90 days after dialysis initiation for patients younger than 65 , but start immediately after renal transplant.

The file SAF.PATIENTS was used as the primary data set, including cause of renal disease (PDIS) and cause and date of patient death. SAF.RXHIST was used to obtain follow-up dates. The file SAF.TXWAIT contains the date patients in the above cohort were first placed on the transplant waiting list. The file SAF.MEDEVID includes data from the Medical Evidence Form (2728). The file SAF.TXUNOS included information on transplant donor type, pre-transplant dialysis, previous transplant, and multiple organ transplants. Files were merged using unique patient identifiers. Details on anthropometric measurements or nutritional parameters other than serum albumin were unavailable. No information on patient medications was available (for the entire cohort of patients) except for the use of pre-dialysis erythropoietin. The USRDS researcher's agreement specifically prohibits patient contact or chart review.

All analyses were performed using SPSS 9.0 TM (SPSS, Inc., Chicago, IL). Files were merged and converted to SPSS files using DBMS/Copy (Conceptual Software, Houston, TX). Statistical significance was defined as $\mathrm{p}<0.05$. Univariate analysis was performed with Chi-square testing for categorical variables and Student's t-test for continuous variables. Variables with $\mathrm{p}<0.10$ in univariate analysis for a relationship with elevated BMI were entered into multivariable analysis as covariates. Continuous variables were examined for outliers, and values $\geq 3$ SD from the mean were removed from analysis with the exception of height and weight, as above, as per prior reports.

Stepwise linear regression was used to calculate the independent association of Polycystic kidney disease, adjusted for other factors, with both Hematocrit and hemoglobin levels. Factors included BMI, race, age, gender, year of first dialysis session, cause of end stage renal disease (diabetes, PKD, or other causes), dialysis type (hemodialysis vs. peritoneal dialysis) and additional variables from the medical evidence form, including diabetes and hypertension as comorbidities distinct from causes of ESRD, and ESRD network, as the independent variables.

Stepwise logistic regression was used to model independent associations with Polycystic kidney disease, using the same variables as in linear regression.

Stepwise Cox Regression non-proportional hazards analysis modeled the association between polycystic kidney disease with all-cause mortality, controlling for the same variables as in linear regression above. Survival analysis compared all patients on the transplant waiting list, censoring patients for receipt of renal transplantation. 


\section{Survival Functions}

\section{By cause of ESRD}

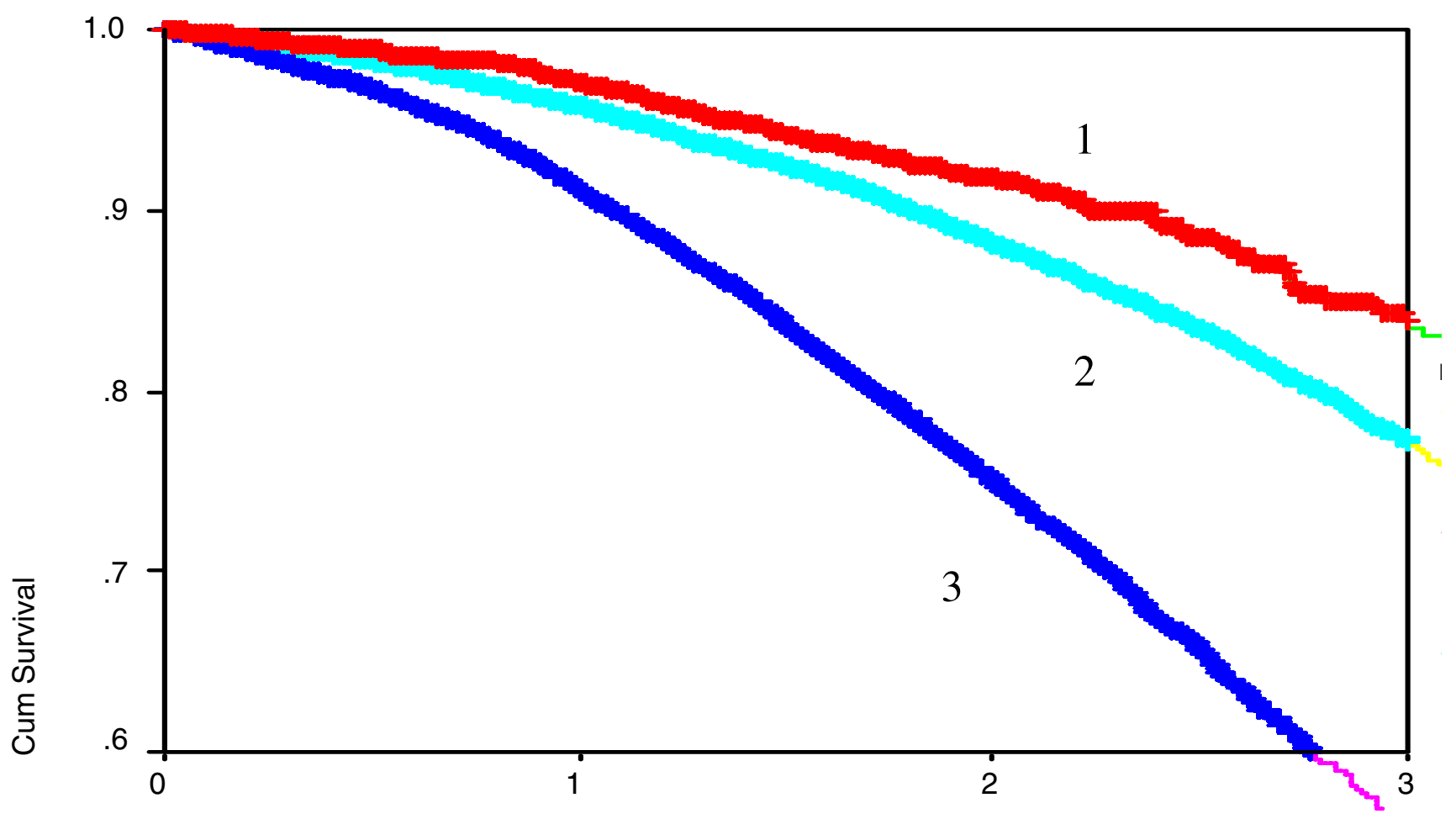

Years after Listing

\section{Figure I}

Unadjusted survival, by cause of ESRD, patients on the renal transplant waiting list. U.S. Patients on the renal transplant waiting list, who started dialysis from I April I 995-29 June 1999, $\mathrm{N}=40,493$. I = Patients with Polycystic kidney disease, $2=$ patients without Polycystic kidney disease or diabetes, $3=$ patients with diabetes. All diseases listed are as causes of end stage renal disease.

Survival time was calculated as the time from the date of the first listing for transplant until death, or latest available follow-up date or receipt of renal transplant. We did not remove patients from the category of listing for transplant if they were removed from this category at a later time, nor did we remove patients from the category of renal transplant recipient if they later experienced graft loss, in intent to treat fashion. Hierarchically well-formed models were used for assessment of interaction terms in all models.

\section{Results}

Of 348,615 patients who initiated ESRD therapy in the USRDS database from 1 April 1995 to 29 June 1999, 43,707 were subsequently entered on the renal transplant waiting list, of whom 40,493 were entered on the waiting list on or after 1 April 1995. Of these, $89 \%$ had sufficient information from the medical evidence form (2728) for hematocrit. The mean date of first ESRD service was 14 January 1997 . The total study cohort had 12,127 recipients of cadaveric kidneys and 4200 recipients of living donor kidneys, in addition to wait-listed patients who did not receive transplants during the study period. The mean transplant date was 25 Dec 1997, and the most recent transplant date was 16 November 1999 . The most recent follow-up date was 29 June 2001. Thus, the study had 51 months of accrual and 24 months of additional followup.

For patients with causes of ESRD other than PKD, median hematocrit in 1995 was $27.2 \pm 5.3 \%$, in $199627.4 \pm 5.4 \%$, 
Table I: Factors assessed in ESRD patients on the renal transplant waiting list who started dialysis on or after I April I995-29 June I999

\begin{tabular}{|c|c|c|c|}
\hline Factor & Wait-Listed Patients & $\begin{array}{l}\text { Univariate Odds Ratio for } \\
\text { Polycystic Kidney Disease }\end{array}$ & $\begin{array}{l}\text { Adjusted Odds Ratio for } \\
\text { Polycystic Kidney Disease In } \\
\text { Logistic Regression ( } 95 \% \\
\text { CI) }\end{array}$ \\
\hline
\end{tabular}

$\begin{array}{ll}\text { N } & 40,493 \\ \text { PKD } & 2949 \\ \text { Male } & 24,143(59.6) \\ \text { African American } & 11,193(27.6) \\ \text { Year of First Dialysis } & 1996.5 \pm 1.1 \\ \text { Mean Age (Years) } & 45.6 \pm 13.9 \\ \text { Mean Followup (Years) } & 1.4 \pm 1.0 \\ \text { Renal Transplantation } & 16,327 \\ \text { Continuous Variables from Medical } & \\ \text { Evidence Form, 2728 (at initiation } & \\ \text { of dialysis): } & \\ \text { Recipient Weight (in kg) } & \\ \left.\text { Body Mass Index (kg/m }{ }^{2}\right) & 75.7 \pm 13.7 \\ \text { Hematocrit (\%) } & 25.8 \pm 4.9 \\ \text { Hemoglobin (gm/dl) } & 28.2 \pm 5.5 \\ \text { Serum Albumin (gm/dl) } & 9.5 \pm 1.9 \\ \text { Serum Creatinine (mg/dl) } & 3.4 \pm 0.6 \\ \text { BUN (mg/dl) } & 10.0 \pm 4.4 \\ \text { Categorical Variables from the } & 94.4 \pm 33.5 \\ \text { Medical Evidence Form (2728), his- } & \\ \text { tory of: } & \\ \text { COPD } & \\ \text { Congestive Heart Failure } & 615(1.5) \\ \text { Ischemic Heart Disease } & 4621(11.4) \\ \text { Myocardial Infarction } & 3179(7.9) \\ \text { Peripheral vascular disease } & 1105(2.7) \\ \text { Diabetes (Primary) } & 1879(4.6) \\ \text { Hypertension } & 8068(22.1) \\ \text { Cancer } & 26,658(73) \\ \text { Alcohol use } & 451(1.1) \\ \text { Pre dialysis EPO } & 373(0.9) \\ \text { Hemodialysis (vs. Peritoneal Dialysis) } & 11,108(30.4) \\ & 28,003(76.7) \\ & \end{array}$

$\begin{array}{ll}0.89(0.83-0.97) & 0.89(0.79-0.99) \\ 0.27(0.24-0.31) & 0.25(0.22-0.30) \\ & 1.02(1.02-1.03) \\ & 0.98(0.97-0.99) \\ 1.48(1.38-1.59) & 1.23(1.10-1.39)\end{array}$

$1.01(1.01-1.02)$

$1.02(1.01-1.03)$

$1.04(1.03-1.05)$

$2.67(2.4 I-2.96)$

$0.97(0.95-0.98)$

$\begin{array}{ll}0.68(0.47-0.97) & 0.60(0.37-0.96) \\ 0.18(0.15-0.23) & 0.31(0.23-0.41) \\ 0.58(0.49-0.69) & 0.69(0.54-0.88) \\ & 1.67(1.17-2.38) \\ 0.19(0.13-0.27) & 0.34(0.22-0.54) \\ 0.07(0.05-0.09) & 0.59(0.44-0.79) \\ & \\ 0.31(0.16-0.61) & 0.41(0.25-0.67) \\ 1.10(1.01-1.20) & 0.42(0.20-0.88) \\ 0.88(0.80-0.96) & \end{array}$

In column one, data given as the number (\% of total) or mean \pm one standard deviation of patients with polycystic kidney disease who had the disease or factor. In column two, data are for all other ESRD patients. Odds ratios are the strength of the association of a disease or factor with polycystic kidney disease, with 1.0 being neutral. ${ }^{*}=p<0.0$ I vs. patients with all other causes of ESRD by Chi Square Test Ap $<0.0$ I vs. patients with all other causes of ESRD by Student's t-test ${ }^{B}$ controlled for all covariates listed in methods section. Only results with statistical significance $(p<$ 0.01 ) are shown.

in $199728.0 \pm 5.4 \%$, in $199828.8 \pm 5.4 \%$, and in 1999 $30.5 \pm 5.4 \%$. There was significant increase in hematocrit by year in linear regression analysis $(\mathrm{p}<0.01)$. For patients with ESRD due to PKD, median hematocrit in 1995 was $29.9 \pm 5.7 \%$, in $199629.6 \pm 5.3 \%$, in $199730.5 \pm$ $5.4 \%$, in $199830.9 \pm 5.6 \%$, and in $199931.3 \pm 5.5 \%$. There was also a significant increase in hematocrit by year for these patients in linear regression analysis $(\mathrm{p}<0.01)$.

Table 1 shows descriptive statistics of the study population, which were comparable to previous USRDS reports. The far right column shows results of logistic regression analysis of factors independently associated with polycystic kidney disease.

Figure 1 shows unadjusted survival stratified by patients with ESRD due to Polycystic kidney disease, diabetes, and all other causes (excluding Polycystic kidney disease and diabetes). Polycystic kidney disease was associated with statistically significant reduced mortality ( $\mathrm{p}<0.001$ by Log Rank Test) vs. either diabetics or non-diabetics. The hazard ratio for patients with Polycystic kidney disease was 0.64, 95\% CI 0.53-0.77, p < 0.001 in Cox Regression. Figure 3 shows a survival plot of patients with Polycystic kidney disease vs. all other ESRD patients, excluding those 
with ESRD due to diabetes. Compared to non-diabetic ESRD patients, patients with Polycystic kidney disease had a hazard ratio for mortality of $0.68,95 \%$ CI $0.56-0.82$, p $=0.001$.

\section{Discussion}

The present study confirms an association between elevated hematocrit, hemoglobin, and Polycystic kidney disease in a cohort of ESRD disease patients on the renal transplant waiting list. The association between increased hematocrit and polycystic kidney disease was initially noted in small observational studies, which noted higher erythropoietin levels and reticulocyte counts in patients with Polycystic kidney disease as well. [5,6] and later confirmed in registry studies of peritoneal dialysis patients. [7] Although other authors have noted an increase in hematocrit with increasing time on dialysis, [8] the present study was only able to measure hematocrit at the start of dialysis, prior to usual initiation of erythropoietin, although a substantial number of wait-listed patients received pre-dialysis erythropoietin. Regardless, the study again confirms that the survival advantage of Polycystic kidney disease was independent of hematocrit or hemoglobin at the start of dialysis, and in fact was independent of all measured factors. The present study is in agreement with previous reports of positive outcomes of peritoneal dialysis in patients with polycystic kidney. $[1,9,10]$

As we have previously reported, the reasons for the comparatively good survival experienced by patients with polycystic kidney disease are unclear, since these patients have known extrarenal manifestations that may impact longterm survival. While these extrarenal manifestations are emphasized in renal textbooks and training exams, they do not appear to be as significant as the extrarenal manifestations (ie, comorbidity) associated with other diseases causing ESRD, predominantly diabetes and hypertension, as shown in Table 1.

In contrast to our previous study, the present study found that hematocrit was significant in multivariate as well as univariate analysis of factors associated with Polycystic kidney disease. In contrast, the present study did not find significant negative associations between Polycystic kidney disease and either cerebrovascular accident, use of pre-dialysis erythropoietin, or hemodialysis, as was noted in our previous analysis. This is most likely due to changes in population characteristics (the previous study included all patients with end stage renal disease) and the much more complete proportion of patients with evidence from the CMS Form 2728 in the present study (100\%). Howev$\mathrm{er}$, it is notable that hemodialysis was still not more commonly used in Polycystic kidney disease, in contrast to general recommendations, [11] although this recommendation has not been universal. [12] Peritoneal dialysis has been a more frequent modality in patients with polycystic kidney disease than in patients with other causes of ESRD in the last several USRDS reports. However, this association has not previously been demonstrated corrected for age and other factors.

Table 2 shows results of linear regression analysis of factors associated with Hematocrit, while Table 3 shows results of linear regression analysis of factors associated with hemoglobin.

Table 2: Linear regression analysis of factors associated with hematocrit level

\begin{tabular}{lll}
\hline Factor & Standardized Coefficient (Beta) & P Value \\
\hline & & \\
Polycystic Kidney Disease & 0.062 & $<0.00$ I \\
Pre-Dialysis EPO use & 0.123 & $<0.00$ I \\
Serum Albumin & 0.157 & $<0.00$ I \\
Male & 0.113 & $<0.00$ I \\
Age (per year) & 0.034 & $<0.00$ I \\
Year of First ESRD Service & 0.06 & $<0.00 \mathrm{I}$ \\
Smoking & 0.015 & 0.007 \\
Ischemic Heart Disease & 0.013 & 0.027 \\
Hemodialysis (vs. peritoneal dialysis) & -0.076 & $<0.001$ \\
Serum Creatinine (mg/dl) & -0.296 & $<0.001$ \\
Congestive Heart Failure & -0.029 & $<0.001$
\end{tabular}

Dialysis modality was obtained from the Medical Evidence form (HCFA 2728), and was therefore only available for patients who started on ESRD therapy on or after 1995. Only variables that were significant in univariate analysis are shown. 
The limitations of the current study are similar to those of other retrospective studies. Variables not available in the USRDS were as stated in the methods section. Residual confounding may have persisted beyond the ability of statistical adjustment to correct. We were unable to follow laboratory values, particularly hematocrit, over time. Radiographic findings could not be confirmed. The USRDS did not distinguish between PKD1 and PKD2 or other genetic presentations of autosomal dominant polycystic kidney disease. The limitations of the CMS Form 2728 have been reviewed, [4] but the accuracy of this form is greatest for cardiovascular disease, which is the leading causes of death in the dialysis population. The population-based nature of this study minimized issues of selection and center bias. Our comparison of Polycystic kidney disease patients with other patients on the renal transplant waiting list, in addition to information on comorbidity and laboratory data, minimized issues of selection bias as much as a registry study is likely to be able. However, this very selection introduces problems with generalizability, and the results of the present study apply to patients on the renal transplant waiting list, not all patients with end stage renal disease.

In summary, the mean hematocrit of patients with polycystic kidney disease was significantly higher at presentation to ESRD than for patients with other causes of ESRD on the renal transplant waiting list, despite a significant overall increase in such levels in more recent years of the study. The survival advantage of patients with polycystic kidney disease compared to patients with other causes of ESRD was independent of other factors, including renal transplantation.

\section{Competing Interests}

None declared.

\section{Author's Contributions}

KA conceived the research plan, conducted primary analysis of the USRDS datafiles, and was responsible for primary preparation of the manuscript. LA, as an expert on the USRDS, supervised the research plan, analysis, and approved the final version of the manuscript.

All authors read and approved the final manuscript.

\section{Authors's Note}

The opinions are solely those of the authors and do not represent an endorsement by the Department of Defense or the National Institutes of Health. This is a U.S. Government work. There are no restrictions on its use.

\section{References}

I. Perrone RD, Ruthazer R, Terrin NC: Survival after end-stage renal disease in autosomal dominant polycystic kidney disease: contribution of extrarenal complications to mortality. Am J Kidney Dis 2001, 38:777-84

2. Fourtounas $C$, Panteris V, Valis D: Survival after end-stage renal disease in autosomal dominant polycystic kidney disease. Am J Kidney Dis 2002, 39:660

3. Abbott KC, Agodoa LY: Polycystic kidney disease at end-stage renal disease in the United States: patient characteristics and survival. Clin Nephrol 2002, 57:208-I 4

4. Longenecker JC, Coresh J, Klag MJ, Levey AS, Martin AA, Fink NE, Powe NR: Validation of comorbid conditions on the end-stage renal disease medical evidence report: the CHOICE study. Choices for Healthy Outcomes in Caring for ESRD. J Am Soc Nephrol 2000, I I:520-529

5. Chandra M, Miller ME, Garcia JF, Mossey RT, McVicar M: Serum immunoreactive erythropoietin levels in patients with polycystic kidney disease as compared with other hemodialysis patients. Nephron 1985, 39(I):26-9

6. Rotellar C, Gelfand MC: Polycystic kidneys do maintain good endocrine function. Med Hypotheses 1989, 30:6 I-4

7. Linblad AS, Nolph KD: Hematocrit values in the CAPD/CCPD population: a report of the National CAPD Registry. Perit Dial Int 1990, 1 0:275-8

8. Glicklich D, Kutcher R, Rosenblatt R, Barth RH: Time-related increase in hematocrit on chronic hemodialysis: uncertain role of renal cysts. Am J Kidney Dis 1990, I 5:46-54

9. Hadimeri H, Johansson AC, Haraldsson B, Nyberg G: CAPD in patients with autosomal dominant polycystic kidney disease. Perit Dial Int 1998, 18:429-32

10. Locatelli AJ, Marcos GM, Gomez MG, Alvarez SA, DeBenedetti LC: Comparing peritonitis in continuous ambulatory peritoneal dialysis patients versus automated peritoneal dialysis patients. Adv Perit Dial 1999, I 5: 193-6

II. Lieske JC, Toback FG: Autosomal dominant polycystic kidney disease. J Am Soc Nephrol 1993, 3:1442-50

12. Brady HR, Wilcox CS, eds: Renal Cystic Diseases in Therapy in Nephrology and Hypertension (Edited by: Brenner BM Editor in Chief) W.B. Saunders Company, Philadelphia 367-374

\section{Pre-publication history}

The pre-publication history for this paper can be accessed here:

http://www.biomedcentral.com/1471-2369/3/7/prepub
Publish with BioMed Central and every scientist can read your work free of charge

"BioMedcentral will be the most significant development for disseminating the results of biomedical research in our lifetime." Paul Nurse, Director-General, Imperial Cancer Research Fund

Publish with BMC and your research papers will be:

- available free of charge to the entire biomedical community

- peer reviewed and published immediately upon acceptance

- cited in PubMed and archived on PubMed Central

- yours - you keep the copyright

Submit your manuscript here:

http://www.biomedcentral.com/manuscript/ 